\title{
Regeneración de los defectos periodontales intraóseos: combinación de membranas barrera y material de relleno: evidencia preclínica y clínica
}

\section{Regenerative procedure for intra-bony periodontal defects: Combination of barrier membrane and filling material, clinical and preclinical evidence}

\author{
DISCEPOLI N* \\ COSTA BERENGUER X* \\ BASCONES-MARTÍNEZ A**
}

\begin{abstract}
Discepoli N, Costa Berenguer X, Bascones-Martínez A. Regeneración de los defectos periodontales intraóseos: combinación de membranas barrera y material de relleno: evidencia preclínica y clínica. Av Periodon Implantol. 2012; 24, 3: 145-150.
\end{abstract}

\begin{abstract}
RESUIMEN
La terapia periodontal regenerativa tiene como objetivo lo de restaurar los tejidos de soporte periodontal y debería resultar en la formación de nueva inserción conectiva y (nuevo cemento con nuevas fibras del ligamento periodontal insertada) y nuevo hueso. Evidencia histológica en modelos preclínicos ha demostrado regeneración después de tratamiento con membranas barreras, distintos tipos de material de relleno y una combinación de los dos. De todas formas, todavía no queda claro en qué extensión la combinación de membranas barreras y material de relleno puede promover de manera adicional el proceso de regeneración en comparación con tratamientos únicos.
\end{abstract}

PALABRAS CLAVE: Modelos animales, barreras, material de relleno, histología, modelos preclínicos, terapia periodontal regenerativa.

\begin{abstract}
SUMMARY
Regenerative periodontal therapy aims to predictably restore the tooth's supporting periodontal tissues and should result in formation of a new connective tissue attachment (i.e. new cementum with inserting periodontal ligament fibres) and new alveolar bone. Histologic evidence from preclinical models has demonstrated periodontal regeneration following treatment with barrier membranes, various types of grafting materials or a combination thereof. However, it is still not clear to what extenta combination of barrier membranes and grafting materials may additionally enhance the regeneration process compared with barrier membranes alone, grafting materials alone or open flap debridement.
\end{abstract}

KEYWORDS: Animal models, barrier membranes, grafting materials, histology, preclinical models, regenerative periodontal therapy.

Fecha de recepción: 14 de junio de 2009.

Fecha de aceptación: 25 de junio de 2009.

\section{INTRODUCCIÓN}

La regeneración periodontal tiene como principal objetivo restaurar de forma predecible los tejidos de so- porte del diente (nuevo ligamento periodontal, nuevo cemento con fibras conectivas insertadas y nuevo hueso) que habían sido destruidos por enfermedad periodontal o por trauma (Polimeni y cols., 2006). Las te-

* $\quad$ Alumno del Máster de Periodoncia e Implantes. Universidad Complutense de Madrid.

** Catedrático de Medicina Bucal y Periodoncia. Departamento de Estomatología III. Facultad de Odontología. Universidad Complutense de Madrid. 
rapias no quirúrgicas y quirúrgicas convencionales suelen obtener mejoras de las variables clínicas, como reducción de la profundidad de sondaje y ganancia de inserción clínica. Desde el punto de vista histológico estos tipos de terapias acaban con la formación de una inserción epitelial larga sobre la superficie de la raíz tratada, sin la formación de nuevo cemento con fibras del ligamento asociadas (Caton y Greenstein, 1993).

Entre las técnicas más usadas en cirugía periodontal reconstructiva podemos encontrar la que prevé el uso de materiales de distinto origen para rellenar el defecto periodontal (Brunsvold \& Mellonig 1993). Hay evidencia en la literatura que este último tipo de solución lleva a mejoras en términos de variables clínicas como la reducción de la profundidad de bolsa y el nivel de inserción clínica respecto al colgajo de acceso por si sólo (Trombelli 2002, Reynolds 2003).

¿Cuál es la motivación biológica para utilizar esta técnica en cirugía de regeneración? La principal razón biológica está basada en que estos materiales de relleno podrían facilitar la formación de hueso alveolar, ligamento periodontal y cemento radicular a través de uno de los siguientes mecanismos:

1. Contener células capaces de formar hueso (Osteogénesis).

2. Servir como andamio para la formación de hueso (Osteoconducción).

3. Contener sustancias capaces de inducir la formación de hueso (Osteoinducción) (Brunsvold \& Mellonig 1993).

Por otro lado, recientes trabajos en modelos preclínicos han evidenciado que el mecanismo más probable gracias al cual los materiales de relleno juntos con una membrana podrían proporcionar la regeneración ósea y periodontal sería la capacidad de mantener el espacio para el coágulo, más que la osteoconducción (Polimeni, 2004).

A lo largo de las últimas décadas distintos estudios animales han confirmado el importante papel de las células del ligamento periodontal en crear una nueva inserción conectiva ósea, nuevo ligamento periodontal y nuevo cemento con fibras periodontales insertadas (Loe \& Waerhaug 1961; Melcher, 1976; Karring, 1980, 1984, 1985; Nyman, 1980; Lindhe, 1984; Isidor, 1985). Estos estudios, además, han evidenciado que el potencial de las células del ligamento periodontal para formar una nueva inserción conectiva se puede desarrollar sólo si se previene al epitelio y al tejido conectivo de ocupar la zona de la herida al lado de la raíz.
Otras observaciones respecto al tratamiento de defectos intraóseos creados experimentalmente con la implantación de diferentes materiales de relleno o con cirugía de acceso, han evidenciado un tipo de cicatrización caracterizada por la presencia de un epitelio largo de unión hasta la porción más apical de la raíz instrumentada, sin ninguna presencia de regeneración (Caton, 1980). Se ha demostrado que la colocación de una membrana barrera para cubrir el área de la raíz denudada puede excluir el crecimiento del epitelio en la herida periodontal y permite la colonización por parte de las células del tejido conectivo y del hueso alveolar de manera selectiva (Nyman, 1982; Gottlow, 1984). Por otro lado, la cantidad de regeneración obtenible se ve limitada por la cantidad de espacio disponible bajo la membrana. Esto sugiere que la capacidad por un lado de mantener el espacio y por el otro de estabilizar la herida periodontal podrían influenciar grandemente el proceso de cicatrización (Wikesjo \& Nilveus, 1990; Haney, 1993).

Uno de los métodos con el que los clínicos intentan compensar el colapso de la membrana es añadiendo a la misma un material de relleno. De todas formas resultados de estudios clínicos aleatorizados y controlados no soportan claramente el alcance de resultados clínicos superiores en términos de reducción de profundidad de bolsa y de ganancia de inserción clínica al comparar la regeneración tisular guiada sola con la RTG junto con material de relleno (Blumental \& Steinberg, 1990; Chen, 1995; Mellado, 1995; Gouldin, 1996; Paolantonio, 2002; Murphy \& Gunsolley, 2003; Nygaard-Ostby, 2008).

\section{¿CuÁl ES EL EFECTO DE LA COMBINACIÓN DE UNA MEMBRANA Y DE UN MATERIAL DE RELLENO EN EL TRATAMIENTO DE DEFECTOS INTRAÓSEOS EN MODELOS DE ESTUDIOS ANIMALES?}

Para contestar a esta pregunta se han buscado en la literatura estudios con un seguimiento de 4 semanas que evaluasen la cicatrización de defectos intraóseos después del tratamiento con cualquier tipo de membrana y de material de relleno. No se incluyeron en la búsqueda los artículos que tratasen de evaluar sustancias biomiméticas/factores de crecimiento.

Como principales variables respuesta se identificaron:

- Formación de nuevo tejido periodontal: bajo forma de medida lineal o como porcentaje de la raíz instrumentada. 
- Cambio en el tamaño del defecto (evaluado con radiografías o mediante reentrada).

- Cambio en el Nivel de Inserción Clínica (NIC) o de la Profundidad de Bolsa (PB) tras evaluación clínica.

Se han identificado dos estudios que aportan información sobre el resultado del tratamiento de defectos intraóseos con membranas y material de relleno. En el primer estudio (Blumenthal, 2003) (1) se crearon defectos intraóseos de dos y de tres paredes en nueve babuinos adultos. Se permitió la cronificación de los defectos gracias a la colocación de ligaduras durante 8 semanas. Se evaluaron cuatro modalidades de tratamiento:

1. Membrana colágena reabsorbible.

2. Aloinjerto de hueso desproteinizado y congelado (AHDC).

3. Aloinjerto de hueso desproteinizado y congelado (AHDC) + esponja de glicoproteínas.

4. Membrana reabsorbibles +AHDC.

Se evaluaron los resultados clínicos e histológicos 6 meses después del tratamiento. Los resultados evidencian que no hay mucha diferencia en cuanto a las variables clínicas entre los distintos tratamientos en los defectos de tres paredes. De hecho la cantidad de recesión obtenida varía desde 1,17 (tratamiento $n^{\circ} 3$ ) hasta $1,78 \mathrm{~mm}$ (tratamiento $\mathrm{n}^{\circ} 1$ ), con esta diferencia estadísticamente significativa. El NIC varía desde $5,46 \mathrm{~mm}$ (tto. $\mathrm{n}^{\circ} 2$ ) hasta $6,18 \mathrm{~mm}$ (tto. $\mathrm{n}^{\circ} 1$ ). Para esta variable las diferencias resultaron estadísticamente significativas comparando el tratamiento con membrana y los tratamientos con AHDC y AHDC +esponja, ambos a favor de la membrana. No se presentaron diferencias estadísticamente significativas entre membrana sola y membrana con material de relleno. De manera distinta se nota que en los defectos de 2 paredes se obtiene un NIC mayor con el uso de membrana y material de relleno (tto. $n^{\circ}$ 4) respecto al uso solo de membrana (tto. $n^{\circ} 1$ ).

Respecto a las variables histológicas se nota que en los defectos de dos paredes el mayor porcentaje de regeneración se obtiene con el uso de membranas y material de relleno $(43,2 \%)$. En estos defectos el uso de membranas solas resultó en un porcentaje de regeneración del $28.3 \%$.

El segundo estudio revisado (2) comparaba en 4 perros mestizos cuatro modalidades de tratamiento:

1. Control (colgajo de acceso).

2. Membrana de sulfato de calcio (SC).
3. Material de relleno (AHDC).

4. Material de relleno + membrana de SC.

Se crearon defectos intraóseos de tres paredes y se trataron de manera aleatorizada con uno de los cuatro tratamientos propuestos. Las muestras histológicas se obtuvieron 8 semanas después de la cirugía y se analizaron desde el punto histomorfométrico. Se encontró anquilosis en 3 de los 4 perros, y ninguna de las secciones que presentaban AHDC demostró evidencia de actividad ósea metabólica. El uso de membrana de SC o AHDC solos o en conjunto demostró una cantidad de regeneración de hueso y de cemento celular superior al control.

\section{¿CuÁl ES EL EFECTO DE LA COMBINACIÓN DE UNA MEMBRANA Y DE UN MATERIAL DE RELLENO EN EL TRATAMIENTO DE DEFECTOS INTRAÓSEOS EN ESTUDIOS CLÍNICOS?}

Varios estudios clínicos han demostrado que la regeneración tisular guiada (RTG) es una modalidad de tratamiento exitosa en la cirugía periodontal reconstructiva y siendo un procedimiento aceptado en la mayoría de prácticas periodontales. También se ha confirmado histológicamente que hay verdadera regeneración periodontal en defectos intraóseos en humanos tratados con RTG tanto con membranas reabsorbibles como no reabsorbibles. El objetivo al colocar un material de relleno debajo de una membrana es el de mantener el espacio para la regeneración en aquellos casos en los que la membrana no es capaz de mantenerlo por sí sola, evitando así su colapso. Con el fin de evaluar la efectividad de este tratamiento combinado en humanos, se ha realizado una búsqueda bibliográfica de ensayos clínicos en los que se comparara el uso de materiales de injerto combinado con membrana frente a cirugía de acceso o a la colocación de una membrana sola.

\section{RTG + injerto vs cirugía de acceso}

Sculean y cols. (2003) (3) realizaron un estudio clínico controlado para comparar clínicamente el tratamiento de defectos intraóseos profundos con una combinación de xenoinjerto derivado de bovino (XDB) y una membrana de colágeno reabsorbible (grupo test) frente a la cirugía de acceso (grupo control). 28 pacientes, cada uno de los cuales presentaba 1 defecto intraóseo, fueron asignados aleatoriamente a recibir uno de los dos tratamientos, realizándose las mediciones de tejidos blandos en basal y $l$ año tras el tratamiento. Ambos tratamientos presentaron una reducción de la 
profundidad de sondaje (PS) y una ganancia del nivel de inserción clínica (NIC) estadísticamente significativos 1 año después del tratamiento. Sin embargo, el tratamiento test presentó una reducción de la PS ( $\mathrm{p} \leq$ $0,05)$ y una ganancia de NIC $(p \leq 0,001)$ estadísticamente mayor que el grupo control. En el grupo en que se combinó el XDB con una membrana reabsorbible el $100 \%$ de las localizaciones ganaron un mínimo de 3 $\mathrm{mm}$ de NIC, mientras que en el grupo control esto ocurrió en el $29 \%$ de localizaciones.

En un estudio con un diseño a boca partida (Camargo y cols. 2000) (4) se evaluó la efectividad clínica de un mineral óseo poroso bovino en combinación con una membrana de colágeno derivada de porcino (tratamiento test) (Figs. 1 a 4) en la regeneración periodontal de defectos intraóseos en humanos. Se trataron 22 pares de defectos intraóseos y se realizó reentrada quirúrgica 6 meses después. El tratamiento test se comparó con la cirugía de acceso (tratamiento control). Las mediciones clínicas revelaron una reducción significativamente mayor de la profundidad de bolsa (PB) (diferencias de $1,89 \pm 0,31 \mathrm{~mm}$ en vestibular y $0,88 \pm 0,27 \mathrm{~mm}$ en lingual) y una mayor ganancia de NIC en las localizaciones test (diferencias de $1,51 \pm 0,33 \mathrm{~mm}$ en vestibular y de $1,50 \pm 0,35 \mathrm{~mm}$ en lingual). La reentrada quirúrgica mostró un relleno del defecto significativamente mayor a favor de las localizaciones experimentales (diferencias de 2,67 $\pm 0,91 \mathrm{~mm}$ en vestibular y de 2,54 $\pm 0,87 \mathrm{~mm}$ en lingual). La reducción media de $\mathrm{PB}$ en el grupo test fue de 4,03 $\pm 0,94 \mathrm{~mm}$ en vestibular y de 3,14 $\pm 0,90 \mathrm{~mm}$ en lingual. La ganancia media de NIC fue de aproximadamente 3,25 tanto en vestibular como en lingual y los cambios en el relleno del defecto fueron de $3,70 \mathrm{~mm}$.

Tonetti y cols. (2004) (5) en un ensayo clínico aleatorizado multicéntrico también demostraron que la cirugía periodontal regenerativa con RTG/material de sustitución ósea ofrece beneficios adicionales en cuanto a ganancia de NIC, reducción de PB y predictibilidad de los resultados frente a colgajos con preservación de papila solos. De la misma forma Sculean y cols. (2005) (6) también presentaron mejores resultados clínicos de la combinación de xenoinjerto derivado de bovino (BioOss ${ }^{\circledR}$ ) con membrana de colágeno (BioGide PERIO) frente a colgajo de acceso.

\section{RTG + injerto vs RTG}

Se realizó un ensayo clínico aleatorizado (Stavropoulos y cols. 2003)(7) con el fin de evaluar si el Bio-Oss ${ }^{\circledR}$ utilizado conjuntamente con la RTG mejora la cura-

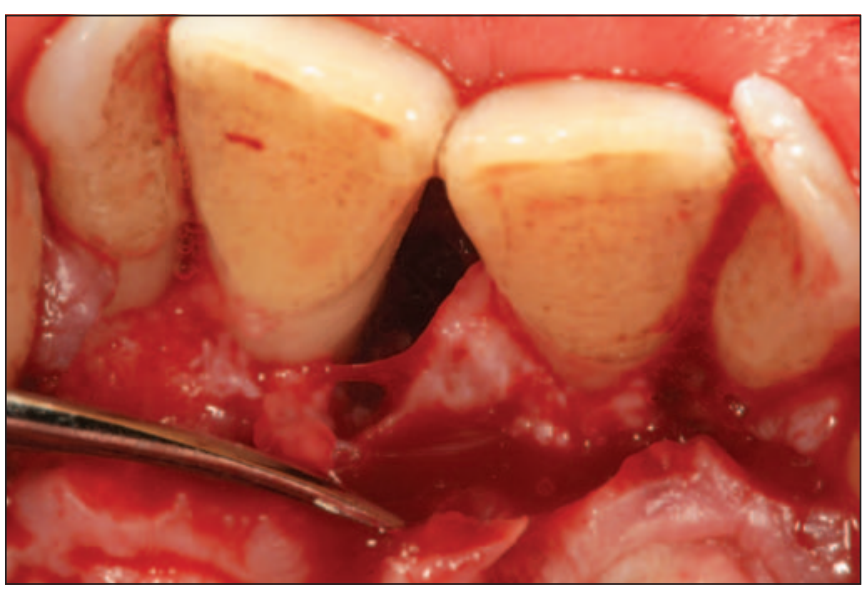

Fig. 1: Defecto intraóseo de dos paredes.

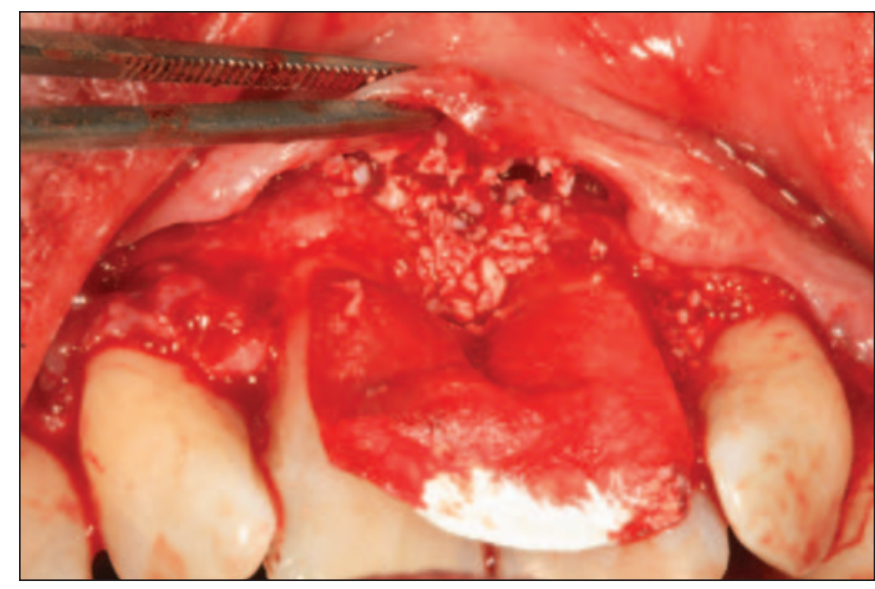

Fig. 2: Relleno con membrana.

ción de defectos intraóseos de 1 o 2 paredes comparándolo con RTG sola. También querían examinar si la impregnación del Bio-Oss $₫$ con gentamicina podía tener un efecto adicional. Para ello se trataron 60 pacientes que presentaban por lo menos un defecto intraóseo interproximal con evidencia radiográfica de componente intraóseo ( $\geq 4 \mathrm{~mm}$ ), asignándolos aleatoriamente a uno de los siguientes tratamientos:

1. RTG con membrana reabsorbible (RTG).

2. RTG con membrana reabsorbible + Bio-Oss $₫$ impregnado con suero salino (DBB-).

3. RTG con membrana reabsorbible + Bio-Oss $\AA$ impregnado con gentamicina (DBB+).

4. Cirugía de acceso.

Todas las modalidades de tratamiento mostraron mejoras clínicas estadísticamente significativas 1 año des- 


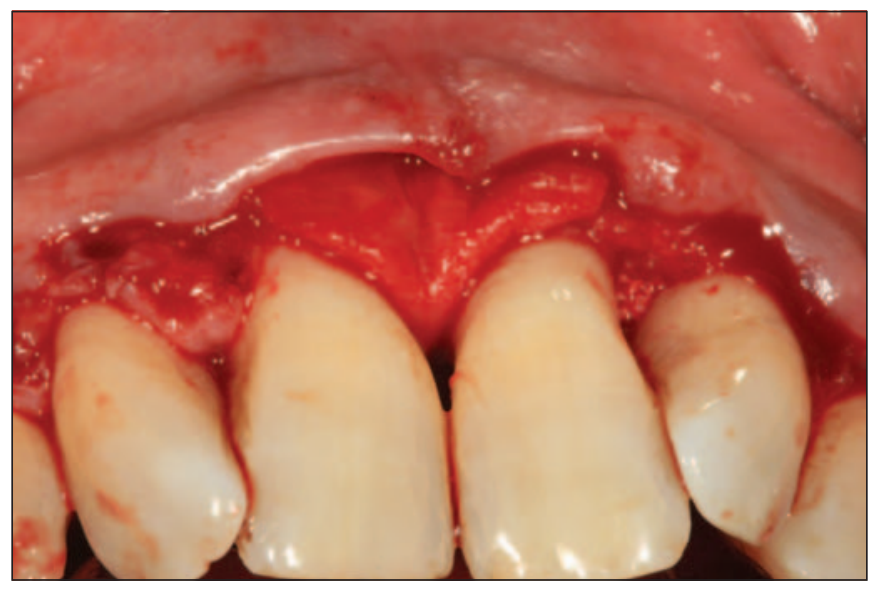

Fig. 3: Recubrimiento con membrana.

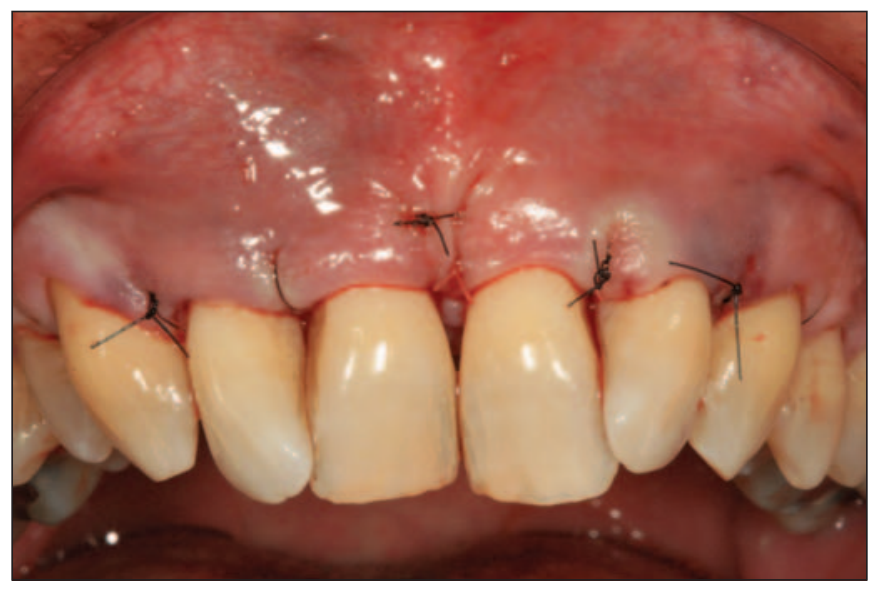

Fig. 4: Sutura y reposición coronal del colgajo.

pués del tratamiento. Los defectos tratados solo con membrana (RTG) mostraron una ganancia en el nivel de inserción al sondaje (NIS) de 2,9 mm, una profundidad de bolsa residual (PB) de 4,9 $\mathrm{mm}$, una ganancia de nivel óseo radiográfico (NOR) de $3,1 \mathrm{~mm}$ y un componente intraóseo (CI) residual de 2,7 mm. La RTG conjuntamente con Bio-Oss $₫$ no aportó mejoras adicionales (ganancia de NIS: $2,5 \mathrm{~mm}, \mathrm{~PB}: 4,9 \mathrm{~mm}$, ganancia NOR: $2,8 \mathrm{~mm}$ y CI: $3,3 \mathrm{~mm}$. Al impregnar el Bio-Oss ${ }^{\circledR}$ con gentamicina $2 \% \mathrm{mg} / \mathrm{ml}$ se observaron mejoras clínicas, superiores a las obtenidas con los otros tratamientos, pero sin alcanzar la significación estadística. Los defectos tratados solamente con cirugía de acceso mostraron las peores respuestas clínicas.

De una forma parecida se evaluó la influencia sobre las variables clínicas (PB, recesión, NIS, placa y sangrado al sondaje), 12 meses después del tratamiento, en función del tipo de membrana utilizada combinándola con un material de injerto (Stavropoulos y cols. 2004) (8). 28 pacientes que presentaban por lo menos 1 defecto intraóseo con una $\mathrm{PB}$ de $\geq 7 \mathrm{~mm}$ y un componente intraóseo radiográfico de $\geq 4 \mathrm{~mm}$ fueron tratados aleatoriamente con una membrana de copolímero de ácido poliláctico/poliglicólico (PLA/PGA) o una membrana de colágeno reabsorbible, ambas en combinación con Bio-Oss $®$. Se observaron mejoras clínicas estadísticamente significativas para ambos grupos 1 año después del tratamiento. Sin embargo, no se observaron diferencias significativas entre los dos tipos de membranas para ninguno de los parámetros evaluados. Para la membrana PLA/PGA la reducción en la PB y la ganancia de NIS fueron de 4,0 $\pm 1,2 \mathrm{~mm}$ y $2,9 \pm$ $2,3 \mathrm{~mm}$ respectivamente, versus $5,1 \pm 1,7 \mathrm{~mm}$ y $3,9 \pm$ $1,3 \mathrm{~mm}$ para la membrana de colágeno. El estudio concluyó que el material de la membrana no es un factor crítico para el resultado de la RTG en el tratamiento de defectos intraóseos con membranas reabsorbibles.

En una revisión sistemática (Murphy y Gunsolley 2003) (9), que tenía por objetivo evaluar la eficacia de los procedimientos de RTG en pacientes con defectos óseos periodontales en comparación con controles quirúrgicos, una de las conclusiones que se obtienen es que la utilización de membranas solas proporciona resultados similares a los obtenidos con la combinación de injertos de sustitución ósea junto con membrana. Esta conclusión se extrae del metaanálisis de 6 ensayos clínicos aleatorizados, sin embargo se puntualiza que ninguno de los 6 estudios registró la individualidad de la anatomía de los defectos tratados, y por ello se cree que la combinación puede ser apropiada en situaciones clínicas específicas.

\section{Histología de RTG + injerto en humanos}

Sculean y cols. (2004) (10) realizaron un estudio para evaluar histológicamente la cicatrización de defectos intraóseos humanos 6 meses después de ser tratados con un xenoinjerto derivado de bovino (XDB) y RTG o un XDB mezclado con colágeno y RTG. Las muestras se obtuvieron de 8 pacientes que presentaban defectos intraóseos muy profundos en dientes programados para extracción. En la mayoría de preparaciones histológicas (7/8) se encontró formación de nuevo cemento con fibras de colágeno insertadas, mientras que en el caso restante la curación de caracterizó por la formación de un epitelio largo de unión a lo largo de la superficie radicular desbridada y no se observó formación de cemento o hueso. En los especímenes que 
mostraban regeneración periodontal, el nuevo cemento siempre fue del tipo celular. En la mayoría de casos las partículas de injerto estaban rodeadas por hueso. En algunas áreas, el tejido óseo alrededor de las partículas de injerto estaba conectado al nuevo cemento de la superficie radicular mediante fibras colágenas insertadas perpendicularmente. El crecimiento epitelial siempre se detenía en la parte más coronal del nuevo cemento formado. No se observaron restos del material de la membrana en ninguna de las biopsias. La encapsulación de las partículas de injerto por tejido conectivo fue poco habitual y estaba limitado a la parte más coronal de los defectos. Los hallazgos de este estudio proporcionan evidencia que el tratamiento de los defectos intraóseos tanto con XDB + RTG o con $\mathrm{XDB}$ colágeno + RTG pueden mejorar la regeneración periodontal en humanos.

\section{CONCLUSIONES}

1. La combinación de una membrana barrera y de materiales de injerto pueden mostrar evidencia histológica de regeneración periodontal, predominantemente reparación ósea.

2. El tratamiento combinado no presenta beneficios adicionales en defectos de 3 paredes.

3. En defectos supraalveolares e intraóseos de 2 paredes, la utilización adicional de materiales de injerto proporcionó resultados histológicos superiores que el uso de membranas solas en cuanto a reparación ósea.

4. En modelos supraalveolares, la combinación de injerto y membrana mostró mejores resultados que el injerto por sí solo.

\section{BIBLIOGRAFÍA}

1. Blumenthal NM, Alves ME, Al-Huwais S, Hofbauer AM, Koperski RD. Defect-determined regenerative options for treating periodontal intrabony defects in baboons. J Periodontol 2003;74:10-24.

2. Kim CK, Kim HY, Chai JK, Cho KS, Moon IS, Choi SH, et al. Effect of a calcium sulfate implant with calcium sulfate barrier on periodontal healing in 3-wall intrabony defects in dogs. J Periodontol 1998;69:982-8.

3. Sculean A, Berakdar M, Chiantella GC, Donos N, Arweiler NB, Brecx M. Healing of intrabony defects following treatment with a bovine-derived xenograft and collagen membrane. A controlled clinical study. J Clin Periodontol 2003;30:73-80.

4. Camargo PM, Lekovic V,Weinlaender M, Nedic M, Vasilic $\mathrm{N}$, Wolinsky LE, et al. A controlled re-entry study on the effectiveness of bovine porous bone mineral used in combination with a collagen membrane of porcine origin in the treatment of intrabony defects in humans. J Clin Periodontol 2000;27:889-96.

5. Tonetti MS, Cortellini P, Lang NP, Suvan JE, Adriaens $P$, Dubravec $\mathrm{D}$, et al. Clinical outcomes following treatment of human intrabony defects with GTR/bone replacement material or access flap alone. A multicenter randomized controlled clinical trial. J Clin Periodontol 2004;31: 770-6.

6. Sculean A, Chiantella GC, Windisch P, Arweiler NB, Brecx M, Gera I. Healing of intra-bony defects following treatment with a composite bovine-derived xenograft (Bio-Oss Collagen) in combination with a collagen membrane (Bio-Gide PERIO). J Clin Periodontol 2005; 32:720-4.

7. Stavropoulos A, Karring ES, Kostopoulos L, Karring T. Deproteinized bovine bone and gentamicin as an adjunct to GTR in the treatment of intrabony defects: a randomized controlled clinical study.J Clin Periodontol 2003;30:486-95

8. Stavropoulos A, Sculean A, Karring T. GTR treatment of intrabony defects with PLA/PGA copolymer or collagen bioresorbable membranes in combination with deproteinized bovine bone (Bio-Oss). Clin Oral Investig 2004;8:226-32.

9. Murphy KG, Gunsolley JC. Guided tissue regeneration for the treatment of periodontal intrabony and furcation defects. A systematic review. Ann Periodontol 2003;8: 266-302.

10. Sculean A, Stavropoulos A, Windisch P, Keglevich T, Karring T, Gera I. Healing of human intrabony defects following regenerative periodontal therapy with a bovine-derived xenograft and guided tissue regeneration. Clin Oral Investig 2004;8:70-4.

\section{CORRESPONDENCIA}

N Discepoli

discepoli_nicola@hotmail.it 